\title{
Keyword Index, Volume 91
}

adaptation 78

admixture 43

AFLP 276, 494

allopatric speciation 239

allopolyploid 268

allozyme electrophoresis 487

allozymes 125, 239, 300, 314, 337, 401, 510

Alnus trabeculosa 331

ancient DNA 9

Anopheles gambiae 407

Anopheles minimus 487

antagonistic pleiotropy 546

aphids 143

Arabidopsis thaliana 36

artificial selection 17

Atlantic cod 557

Austropotamobius pallipes 70

autocorrelation 448

average allele effect 85

Azores islands 239

balanced lethals 584

Bali cattle 565

banteng 565

basidiomycota 163

Bayesian models 117

B chromosomes 577

behaviour 98

bison 565

body pigmentation 440

body size 51, 440

Bombina 136

Bos indicus 43

Bos taurus 248

bottleneck 481

Brassica spp 415

Brevicoryne brassicae 415

cactus hosts 51

candidate gene 60

Castanea 314

centromere identifier 28

chamois 125

Chiapas 415

chloroplast capture 276

chloroplast DNA 465

chromosomal inversion 51

clonal plant 448

clonal reproduction 143

coalescence 107

coevolution 345

Coffea canephora 528

complementary sex determination (CSD) 373

concerted evolution 268

conservation 9

conservation genetics 307

Cornu aspersum 224

Cotesia glomerata 430

cpDNA 276, 389 crown rust resistance 396

Crumenulopsis sororia 193

cutting 331

cyclical parthenogenesis 143

cyt $b 557$

cytoplasmic incompatibility 208, 382

developmental stability 17

diallel 528

dicorynia guianensis 181

directional asymmetry 17

directional selection 78

disease resistance 112

disjunct species divergence time 314

dispersal 153, 422

dispersion 85

distribution 208

disturbance 475

D. melanogaster 202

DNA sequences 481

domestication 43,322

dominance 456

dominance variance 85

doubly uniparental inheritance 354

Drosophila 28, 51

Drosophila melanogaster $78,440,546,570$

Drosophila simulans 202

ecology 345

ecotype 519

effective population size 373

effective size 107

environmental stress 117

epistasis 78, 456

evolution 345

evolutionary rate 239

evolution of sex 78

eye disease 112

Faroe Islands 557

fertility 546

Festuca pratensis 396

fingerprint 519

fish 255

fluctuating asymmetry 17

fluctuating environment 78

forest fragmentation 307

founder event 70

Gadus morhua 557

gallery forest 307

gametophytic lethals 577

gametophytic self-incompatibility 262

gender 60

gene amplification 98

gene conversion 268

gene flow 181, 300, 307, 422, 487

gene markers 337

general combining ability 528

genetic differentiation 70, 181, 415, 510, 519 
genetic distances 36, 494

genetic divergence 314

genetic diversity 153, 300, 307, 322, 401, 519

genetic drift 9

genetic effects 112

genetic load 373

genetic map 293

genetic neighbourhood 448

genetics 255

genetic variability 314

genetic variation 239

genic variance 85

growth hormone axis 60

gymnosperms 584

Gypaetus barbatus 9

habitat differentiation 284

heritability 117, 456

heterosis 36, 456

heterozygosity 401

hierarchical analysis 487

host plant 217

host races 415

hybridisation 276, 354, 465

hybrid zone 136

Hymenoptera 373

inbreeding 85

inheritance 337

insecticide resistance 98

insular endemic 300

interphase 268

introgression 396

islands 407

ITS 284

join-count statistics 173

juvenile growth 60

kangaroos 153

kinship structure 448

$K P$ elements 570

$K P /$ full-sized $P$ elements 570

Labiatae 300

land snail 239

lean growth 494

linkage map 136

lizard 112

local adaptation 415

Lolium multiflorum 396

Madura cattle 565

malaria control 407

male interspecific sexual discrimination 202

management and conservation 401

maternal effects 117

maternal inheritance 510

mating behaviour 502

meiotic segregation 163

mercury 255

metapopulation 107, 345

Mexico 415

microchiroptera 232 microsatellites 9, 43, 136, 143, 153, 181, 217, 284, 361, 407, 422, 448, 475

microspatial differentiation 440

migration 143

mitochondrial DNA 248, 354, 361, 510

molecular diversity 345

molecular markers 322

Moran's I statistics 173

mortality 546

mtDNA 70, 125, 153

mtDNA genealogy 557

multivariate morphometrics 224

mutant traits 17

mutation accumulation 546

Mytilus edulis 354

Mytilus galloprovincialis 354

NAC 173

natural genetics 85

natural population 112

natural selection 440

nrDNA 163

olfactory response 430

Oncorhynchus mykiss 255

Orchidaceae 284

$P$ transposable elements 570

parasitoid 430

paternity skew 373

pathogen evolution 193

Pemphigus bursarius 217

pericentric satellite DNA 232

$\mathrm{pH} 117$

phacochoerus africanus 361

phenotypic plasticity 440

phylogeny 248, 389

phylogeography 361, 389, 465, 510

pigs 494

Pinus sylvestris 193

Pinus taeda L. 584

Pipistrellus pipistrellus 232

Pisum sativum L. 577

plants 577

pleiotropy 98

plio-pleistocene events 224

$\mathrm{P}-\mathrm{M}$ systems 570

point mutation 98

point process 173

population expansion 481

population structure 125, 217, 407

postglacial recolonisation 465

prezygotic selection 584

progenitor germplasm 322

pseudo-overdominance 584

Puccinia coronata 396

QTL 60

QTL analysis 456

quantitative resistance 193

Rana temporaria 117

RAPD 70, 307, 519

RAPD-SSCP 373 
rDNA 268

realized heritability 17,430

reciprocal effects 36

reciprocal transplant 193

recombination 78, 163, 262

recruitment 401

repetitive DNA 232

reproductive isolation 284

reproductive system 276

rescue 28

resistance 345

reticulate evolution 163

RFLP 125, 510

risk assessment 276

Rissa tridactyla 422

Rosaceae 262

Rupicapra rupicapra 125

Ryukyu Islands 300

\section{$S$ allele 502}

Salix caprea 465

Salix viminalis 293

sandalwood 389

Scrophulariaceae 262

seed dispersal 475

selection 401, 430, 494, 519

selfing 584

semiochemicals 430

Senecio 502

senescence 546

sex 143

sex determination 293

small gamodemes 85

SNP 60

Solanaceae 262

South American cattle 248

spatial analysis 181

spatial autocorrelation 173, 331, 422, 475 spatial autocorrelation analysis 502

spatial genetics 173

spatial structure 224, 382

speciation 28,382

species complex 487

species identification 487

spider mite 208

sporophytic self-incompatibility 502

SSCP 136

statistical test 481

stepwise 43

T. cordata 337

temporal method 9

tertiary trisomy 577

T. × europaea hybrids 337

Theobroma cacao L. 322

tick 422

tolerance 255

toxicity 255

T. platyphyllos 337

TP spacer 557

translocations 70, 577

Trichaptum abietinum 163

Trinitario 322

triple test cross 456

tropical trees 181, 307

western mediterranean 224

wing loading 51

wisent 565

Wolbachia 208, 382

Y-chromosome 565

yield stability 528

Zostera marina 448 